\title{
Harvesting the Wind Energy by Triboelectric Nanogenerator for Intelligent High-speed Train System
}

Chuguo Zhang ${ }^{\mathrm{a}, \mathrm{b}, \grave{\dagger}}$, Yuebo Liu ${ }^{\mathrm{a}, \mathrm{d}, \grave{\dagger}}$, Baofeng Zhang ${ }^{\mathrm{a}, \mathrm{b}, \hat{\dagger},}$, Ou Yang ${ }^{\mathrm{a}, \mathrm{b}}$, Wei Yuan ${ }^{\mathrm{a}, \mathrm{b}}$, Lixia He ${ }^{\mathrm{a}, \mathrm{b}}$, Xuelian Wei ${ }^{\mathrm{a}, \mathrm{b}}$, Jie Wang ${ }^{\mathrm{a}, \mathrm{b}, \mathrm{d}^{*}}$, Zhong Lin Wang ${ }^{\mathrm{a}, \mathrm{c}^{*}}$

${ }^{a}$ Beijing Institute of Nanoenergy and Nanosystems, Chinese Academy of Sciences, Beijing 100083, P. R. China

${ }^{\mathrm{b}}$ College of Nanoscience and Technology, University of Chinese Academy of Sciences, Beijing 100049, P. R. China

c School of Materials Science and Engineering, Georgia Institute of Technology, Atlanta, GA 30332, USA

d Center on Nanoenergy Research, School of Physical Science and Technology, Guangxi University, Nanning, 530004, P. R. China

$\uparrow$ These authors contributed equally to this work.

\section{Corresponding Authors}

*Email:wangjie@binn.cas.cn

*Email:zhong.wang@mse.gatech.edu

\section{Author Contributions}

Chuguo Zhang, Yuebo Liu, Baofeng Zhang contributed equally to this work. 


\section{METHODS}

\section{The fabrication of TENGs}

Selection of dielectric materials used for TENG: Firstly, individual dielectric material (PTFE, PVC, FEP) with a thickness of $100 \mu \mathrm{m}$ is attached on acrylic sheets $(40 \mathrm{~mm} \times$ $10 \mathrm{~mm} \times 5 \mathrm{~mm})$. Four acrylic sheets are then fixed on an acrylic board $(60 \mathrm{~mm} \times$ $100 \mathrm{~mm} \times 5 \mathrm{~mm}$ ) with an intervals of $1 \mathrm{~mm}$. Next, the interdigital copper foil with a gap of $1 \mathrm{~mm}(60 \mathrm{~mm} \times 90 \mathrm{~mm} \times 5 \mathrm{~mm})$ is attached to an acrylic board (60 $\mathrm{mm} \times 100 \mathrm{~mm} \times 5 \mathrm{~mm})$ as slider (Figure $\mathbf{S 3})$.

Fabrication of the RS-TENG: The RS-TENG is assembled with a stators and one rotator as shown in Figure S5. The stator of RS-TENG is fabricated by the PCB of ER-TENG coated with film $(100 \mu \mathrm{m})$. The rotator of RS-TENG is fabricated by a disc-shaped PCB board with dimeter of $28 \mathrm{~cm}$, which is used as the supporting substrate. Where a predeposited copper film on the PCB surface was regarded as free-standing triboelectric layer. The commercial PCB has radial-arrayed sectors (12 in all) and the same central angle of about $30^{\circ}$.

Fabrication of the ER-TENG: The ER-TENG is assembled with an electrode as stators, and the other one as rotator as shown in Figure S6. The stator of ER-TENG is only fabricated by electroplating a layer of copper on PCB $(1.6 \mathrm{~mm}$ thick with diameter of $300 \mathrm{~mm}$ ). This copper has been evenly divided into 24 parts with a gap of $1 \mathrm{~mm}$ and 12 alternative parts are connected by inner ring and outer ring respectively to form two electrodes. The rotator of ER-TENG is fabricated with acrylic sheets (2.6mm thick) with the diameter of $300 \mathrm{~mm}$. Then, the 12 fan rings consisting of 
PTFE film $(100 \mu \mathrm{m})$ and kapton $(150 \mu \mathrm{m})$ as shown in Figure S1 are symmetrically and evenly fixed on the circular rotator. Once the direction of PTFE and kapton film bending is determined, the rotator can rotate towards one direction. Rotator is fixed to motor shaft by a flange-mount shaft collar.

Fabrication of double-layer ER-TENG: The fabrication process of the double-layer ER-TENG can be divided into the preparation of the ER-TENG1 and upper ER-TENG2. The ER-TENG2 is assembled with two same electrodes as stators, and one rotator as shown in Figure 1b. The stator is fabricated by electroplating a layer of copper on PCB (1.6mm thick with diameter of $300 \mathrm{~mm})$. This copper has been evenly divided into 24 parts with a gap of $1 \mathrm{~mm}$ and 12 alternative parts are connected by inner ring and outer ring respectively to form two electrodes. The rotator is fabricated with acrylic sheets $(2.6 \mathrm{~mm}$ thick) with the diameter of $300 \mathrm{~mm}$. Then, the 12 fan rings consisting of PTFE film $(100 \mu \mathrm{m})$ and kapton $(150 \mu \mathrm{m})$ as shown in Figure S1 are symmetrically and evenly fixed on the circular rotator. Notably, the total area of dielectric material is $16 \mathrm{~cm}^{2}$. Once the direction of PTFE and kapton film bending is determined, the rotator can rotate towards one direction. Rotator is fixed to motor shaft by a flange-mount shaft collar. The ER-TENG1 is as same as the ER-TENG2.

Fabrication of windmill : The windmill made by 3D-print with the resinous material consists of a shaft with the diameter of $5 \mathrm{~cm}, 7$ spokes with the length of $16 \mathrm{~cm}$ and 7 fan blades with the height of $22 \mathrm{~cm}$ (Figure S15). 


\section{Electrical measurement of the TENGs}

The transferred charges and short-circuit current are tested by programmable electrometer (Keithley model 6514). The pen-circuit voltage is measured by domain oscilloscope (MDO3024), the voltage of the capacitor in charging capacitor test is recorded by a potentiostat (Biologic, VMP3) with the employment of the linear motor (E1100) and rotating motor (BXS6400C). The rotation motor is used to provide different operating conditions for TENG. 


\section{Supplementary Notes}

\section{Supplementary Note 1: The dimensional design of PTFE geometry.}

In order to ensure a good geometry contact, the ER-TENG is designed as an elastic structure with symmetrical top and bottom (Figure S1a). In addition, because the electrode unit of the generator is a fan ring structure with the angle of $\theta$, radium of $R$ and outer radium of $\mathrm{L}$, the PTFE is cut into a fan-shaped structure with the angle of $4 \theta$, radium of R and side length of L (Figure S1b and Figure S1c). The fan ring with an angle of $\theta$ between the two edges of PTFE are used to contact with the copper electrode of upper and lower fan ring as the triboelectric material, and the middle part is applied to provide certain rigidity to realize the elastic function.

\section{Supplementary Note 2: The parameter condition of ER-TENG unit under different state by COMSOL simulation.}

In order to better understanding the mechanism of ER-TENG with the electricity generation, based on the corresponding size and parameters of ER-TENG, the COMSOL software is used to simulate the $2 \mathrm{D}$ electric potential distribution of ER-TENG units under the different state. The size of two copper electrodes is $37 \mathrm{~mm}$ long and $100 \mu \mathrm{m}$ thick and the gap between them is $1 \mathrm{~mm}$. The dielectric material size of PTFE film is $37 \mathrm{~mm}$ long and $100 \mu \mathrm{m}$ thick, and its surface charge density is $50 \mu \mathrm{C}$ $\mathrm{m}^{-2}$. In addition, deriving from the elastic structure, the PTFE film and copper 
electrode can be completely contacted under low pressure. It is easy to find that the two copper electrodes have the different electric potential and generate the relative electric potential difference under different states. Therefore, the electric energy will generate with the sliding of PTFE film.

\section{Supplementary Note 3: Relationship between the transferred charges of unit} tension and energy harvesting efficiency.

Let $w, v, t, F$ and $E_{1}$ represent the width, motive speed, motive time, driving force and input mechanical energy of TENG in an operative period and they are all fixed constants. Based on the corresponding researches and laws of physics, we can obtain the following formula:

$$
\begin{gathered}
E_{1}=F v t \\
V=k Q \\
I=\sigma w v \\
E_{2}=k \sigma w v Q
\end{gathered}
$$

where $V, I, Q, \sigma$, and $E_{2}$ represent the open-circuit voltage, short-circuit current, transferred charges, surface charges density and converted electrical energy of TENG in an operative period; $k$ represents proportionality coefficient. The energy conversion efficiency has the following relation.

$$
\eta=\frac{k \sigma w}{t} \bullet \frac{Q}{F}
$$

Therefore, it is obvious that the energy harvesting efficiency improves with the transferred charges of unit tension. 
Supplementary Note 4: Relationship between the peak value and root mean square (RMS) values.

For alternating current, the RMS values of current and voltage have the following relationship with peak values:

$$
\begin{gathered}
R_{\mathrm{ms}}(\mathrm{I})=\frac{\int_{t_{1}}^{t_{2}} \mathrm{I}(\mathrm{t})}{\mathrm{t}_{2}-\mathrm{t}_{1}} \\
R_{\mathrm{ms}}(\mathrm{V})=\frac{\int_{t_{1}}^{t_{2}} \mathrm{~V}(\mathrm{t})}{\mathrm{t}_{2}-\mathrm{t}_{1}}
\end{gathered}
$$

where $R_{m s}(I)$ and $R_{m s}(V)$ are the RMS values of current and voltage, $I(t)$ and $V(t)$ are the changing values of current and voltage with time, $t$ is the relative time, $t_{1}$ and $t_{2}$ are the start and termination time of the corresponding calculation. 


\section{Supplementary Figures}

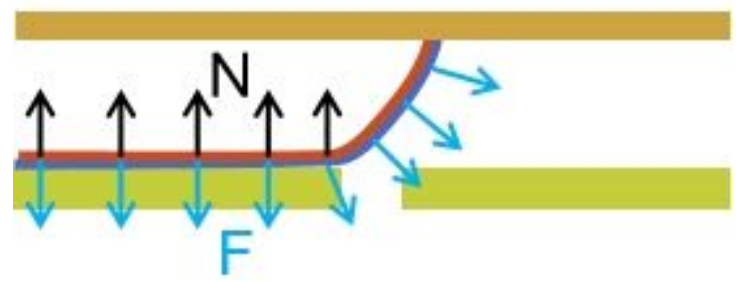

Supplementary Figure 1 Stress analysis diagram of electrification layer of elastic structure.

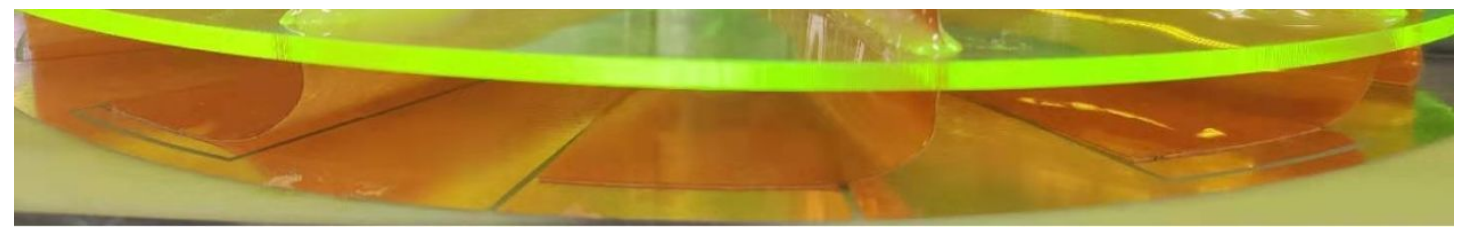

Supplementary Figure 2 The real photo of electrification layer of elastic structure.

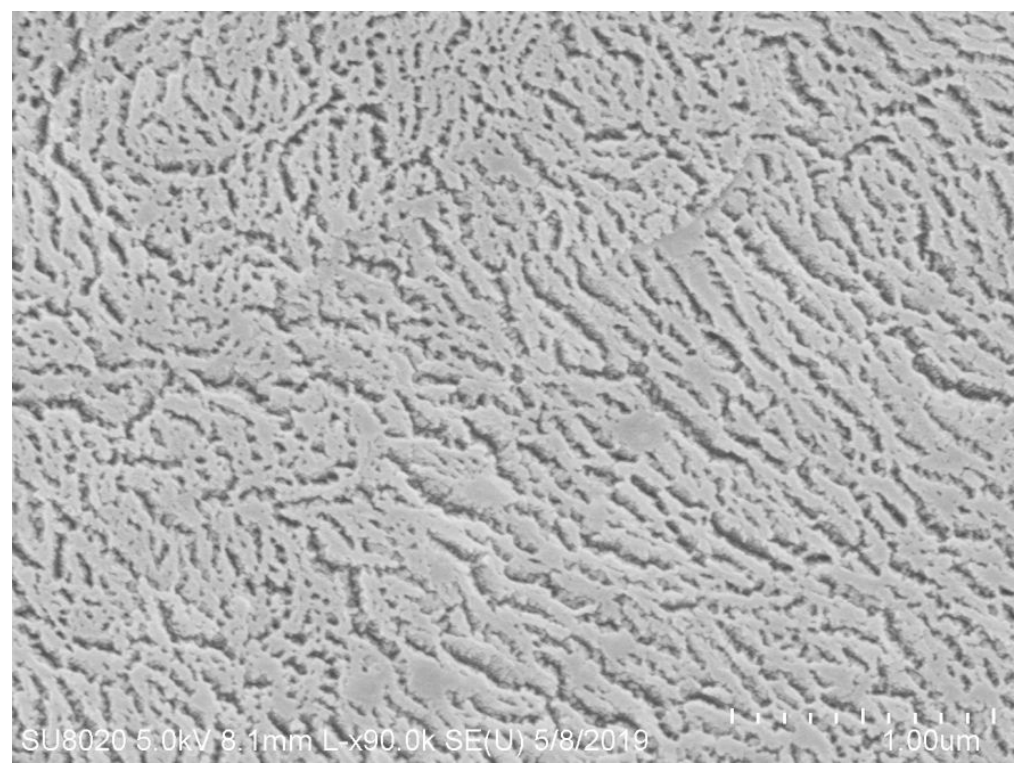

Supplementary Figure 3 The scanning electron microscope (SEM) image of PTFE surface. 


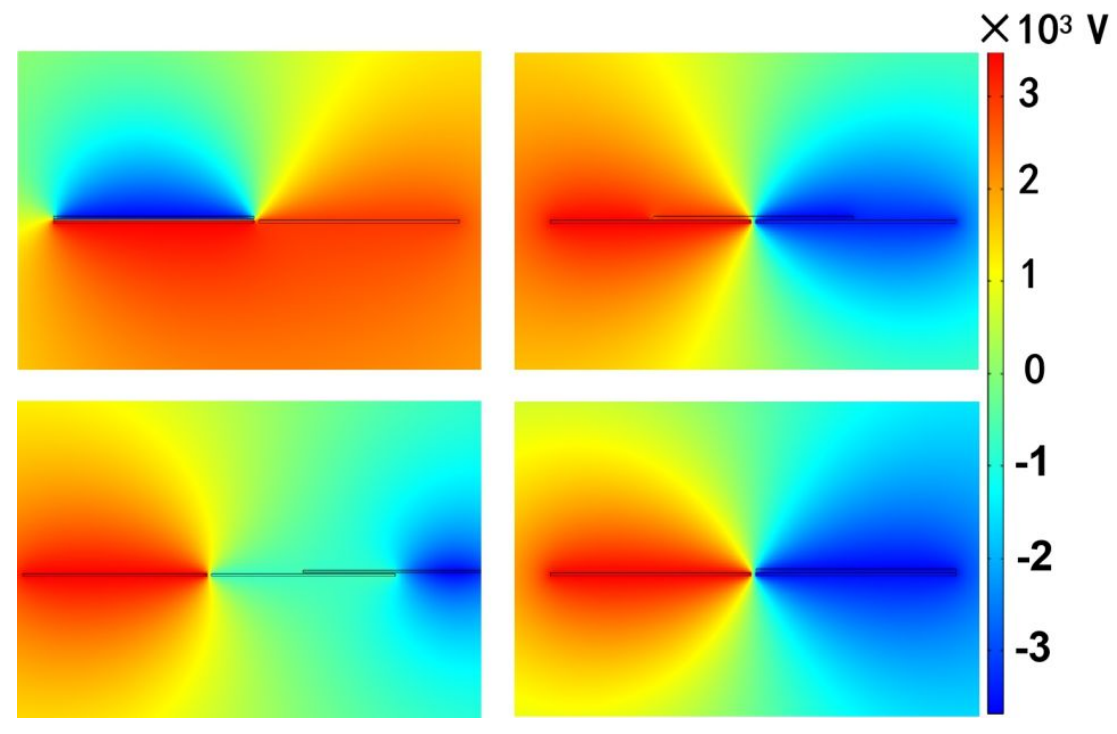

Supplementary Figure 4 The electric potential distribution of ER-TENG unit under different state by COMSOL simulation.

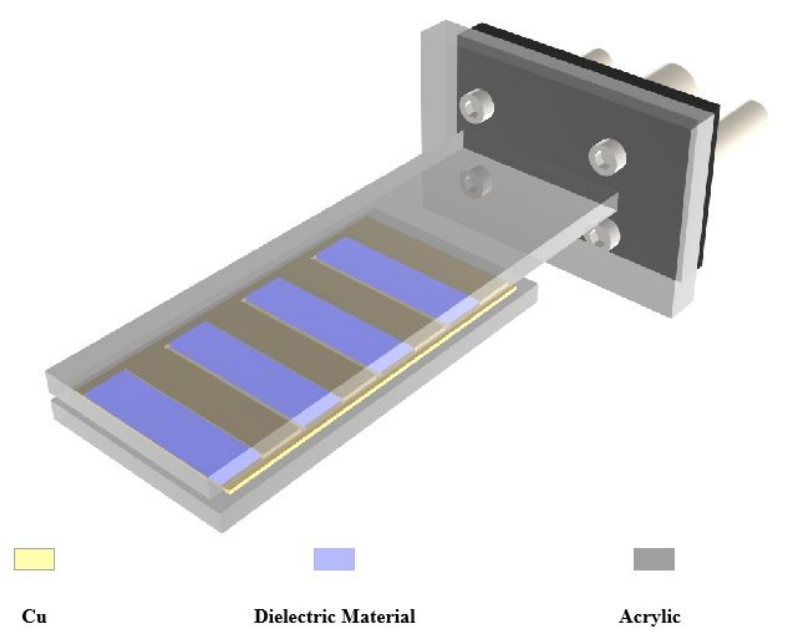

Supplementary Figure 5 The device diagram of TENG for dielectric material selection. 


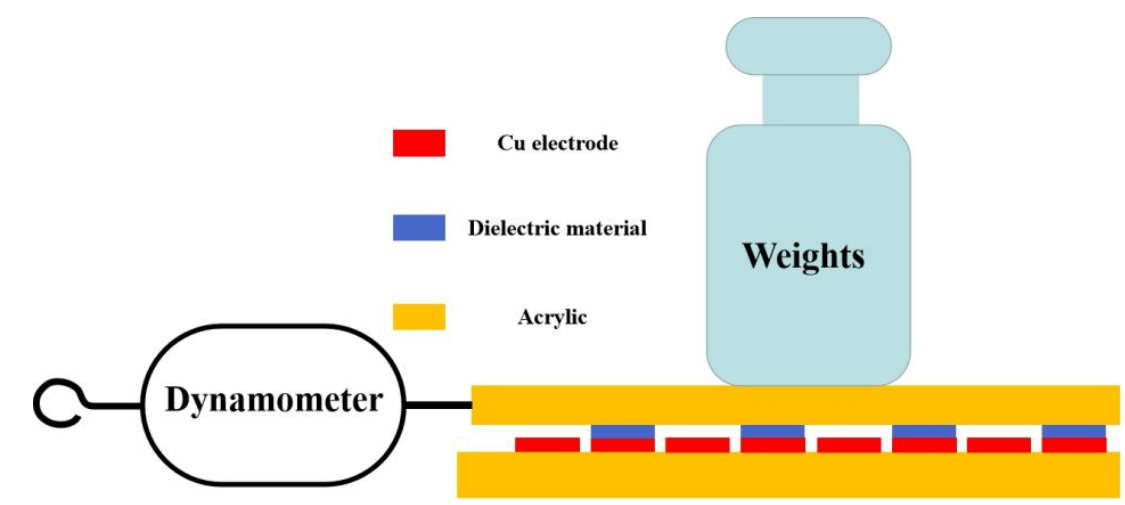

Supplementary Figure 6 The schematic diagram of TENG friction force test.

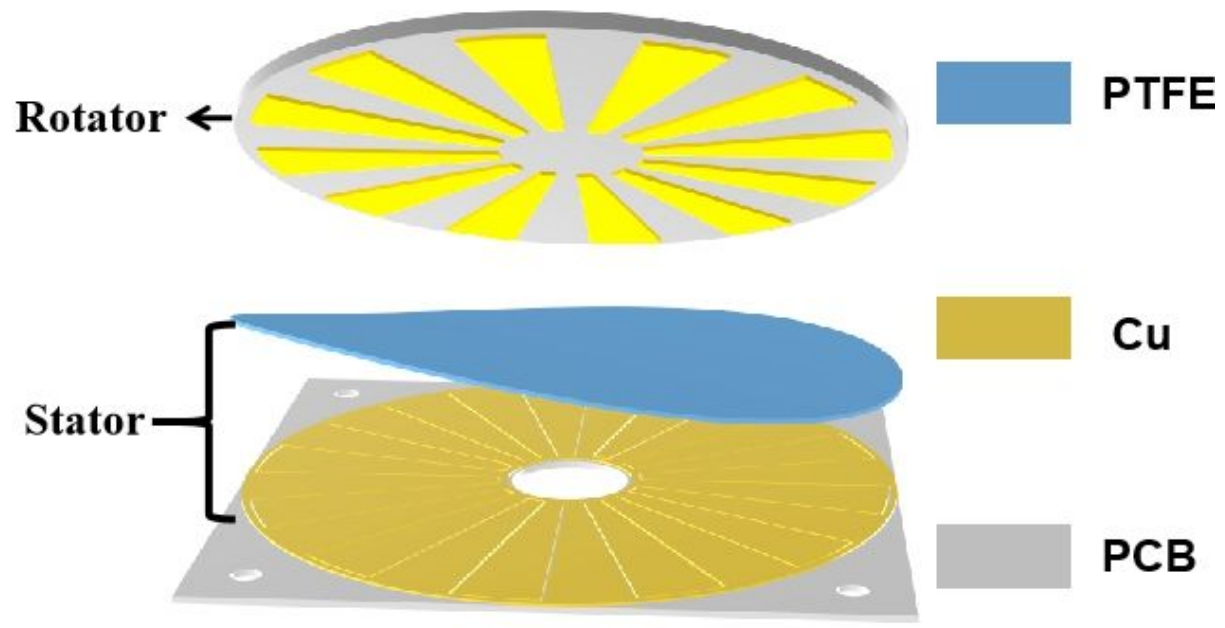

Supplementary Figure 7 The structure diagram of RS-TENG.

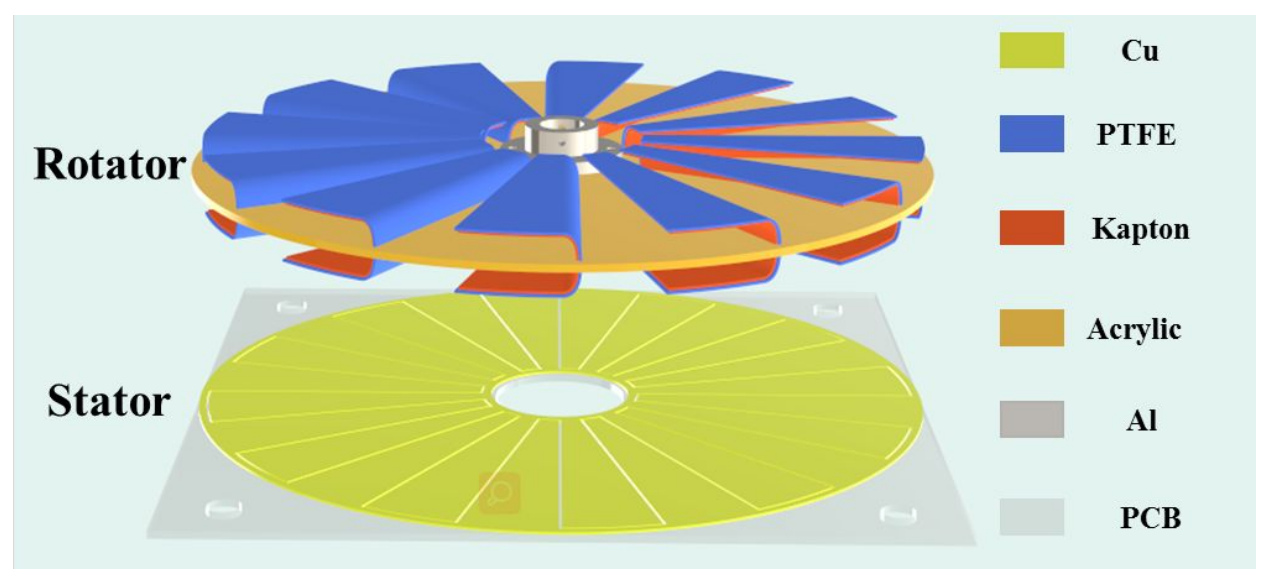

Supplementary Figure 8 The structure diagram of ER-TENG. 


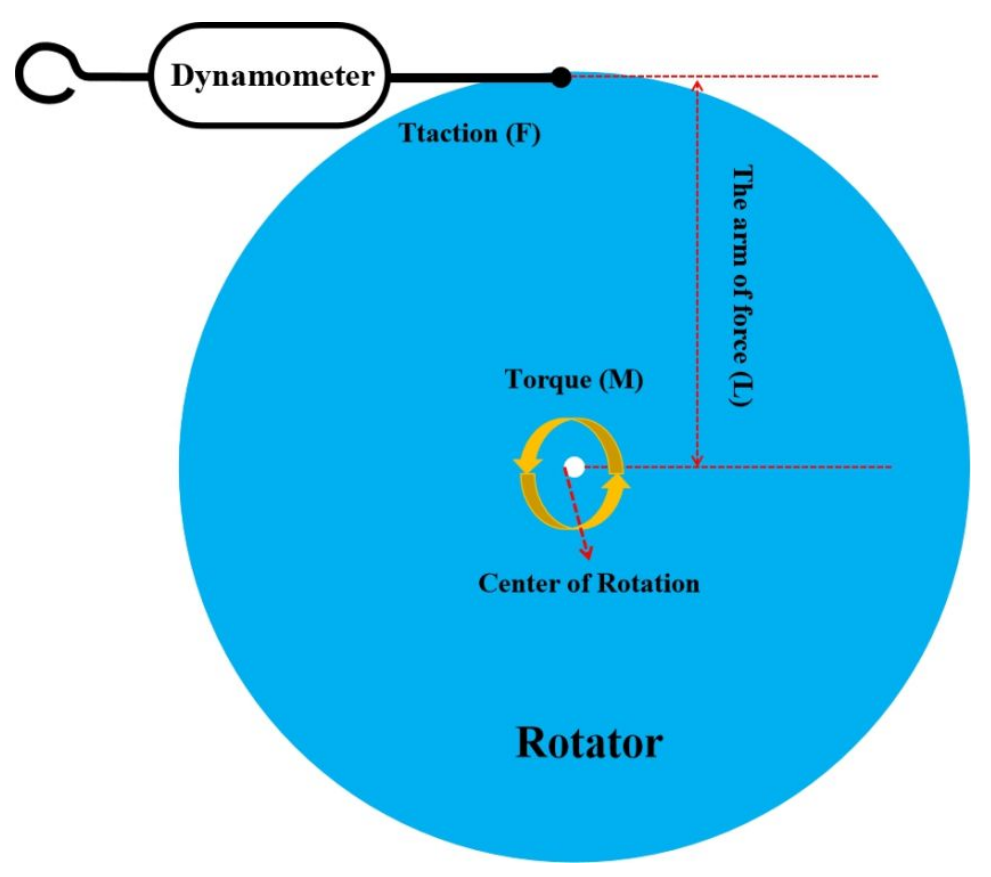

Supplementary Figure 9 The schematic diagram of torque test.

(a)

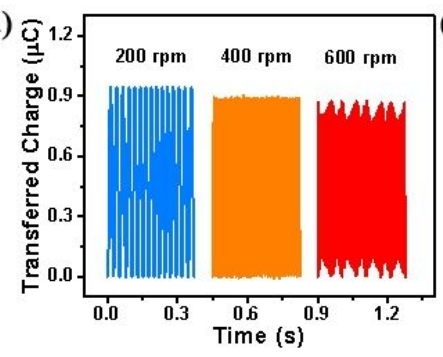

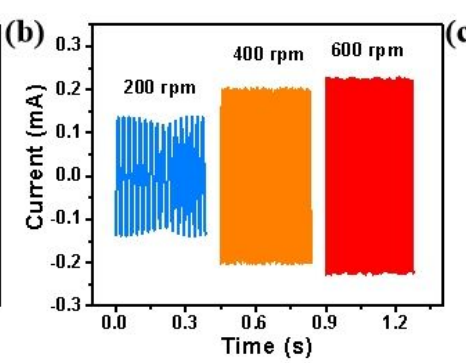

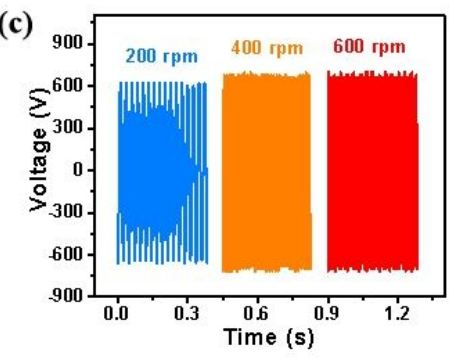

Supplementary Figure 10 The output performance of ER-TENG1 under different rotation speeds. (a) Transferred charges, (b) short-circuit current and (c) open-circuit voltage of ER-TENG1 with different rotation speed of motor. 

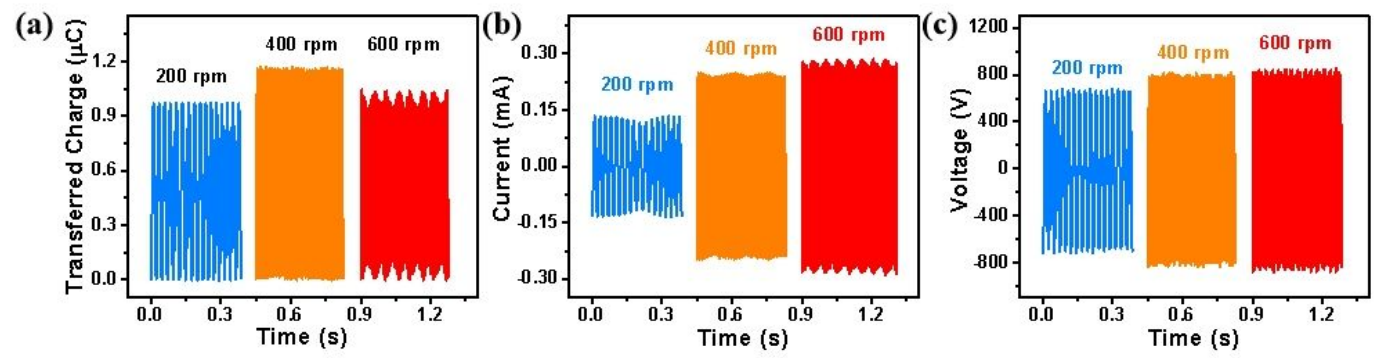

Supplementary Figure 11 The output performance of ER-TENG2 under different rotation speeds. (a) Transferred charges, (b) short-circuit current and (c) open-circuit voltage of ER-TENG2 with different rotation speed of motor.

(a)
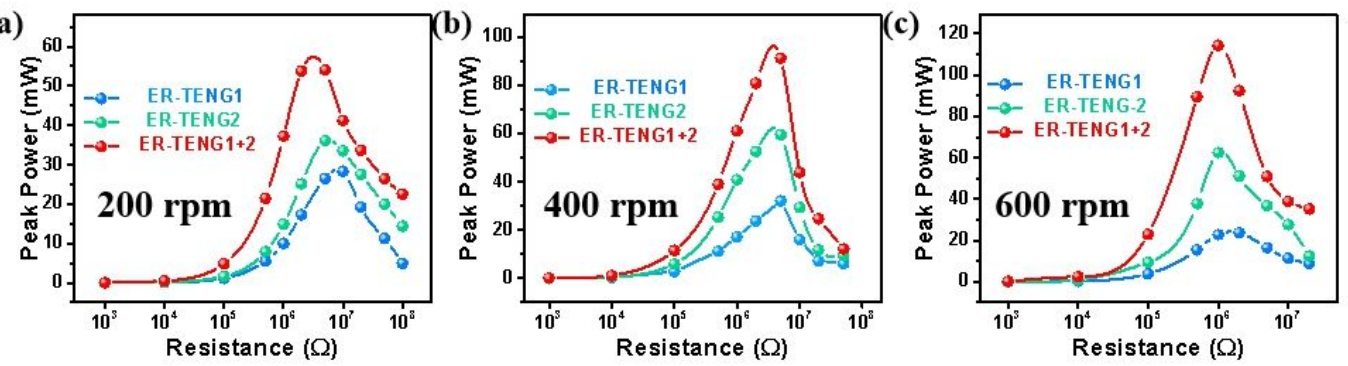

Supplementary Figure 12 The peak power of double-layer ER-TENG, ERTENG1 and ER-TENG2 under different test condition. The peak power resistance profiles of double-layer ER-TENG, ER-TENG1 and ER-TENG2 under the rotation speed of (a) $200 \mathrm{rpm}$, (b) $400 \mathrm{rpm}$ and (c) $600 \mathrm{rpm}$. 


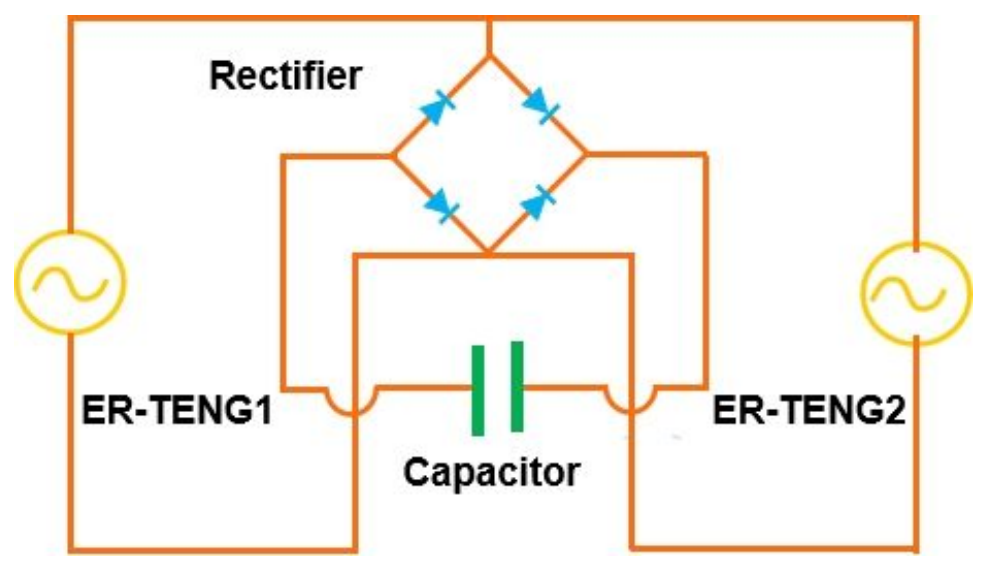

Supplementary Figure 13 The corresponding circuit diagram of double-layer ER-TENG to charge capacitors.
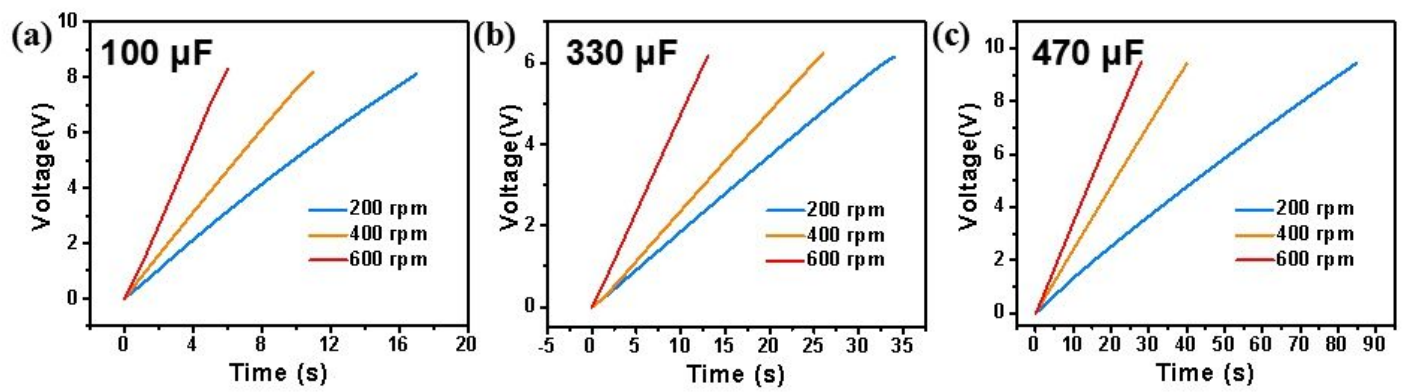

Supplementary Figure 14 The charging curves of capacitors of (a) $100 \mu \mathrm{F}$, (b) 330 $\mu \mathrm{F}$ and (c) $470 \mu \mathrm{F}$ by the ER-TENG1 under different rotation speed of motor.
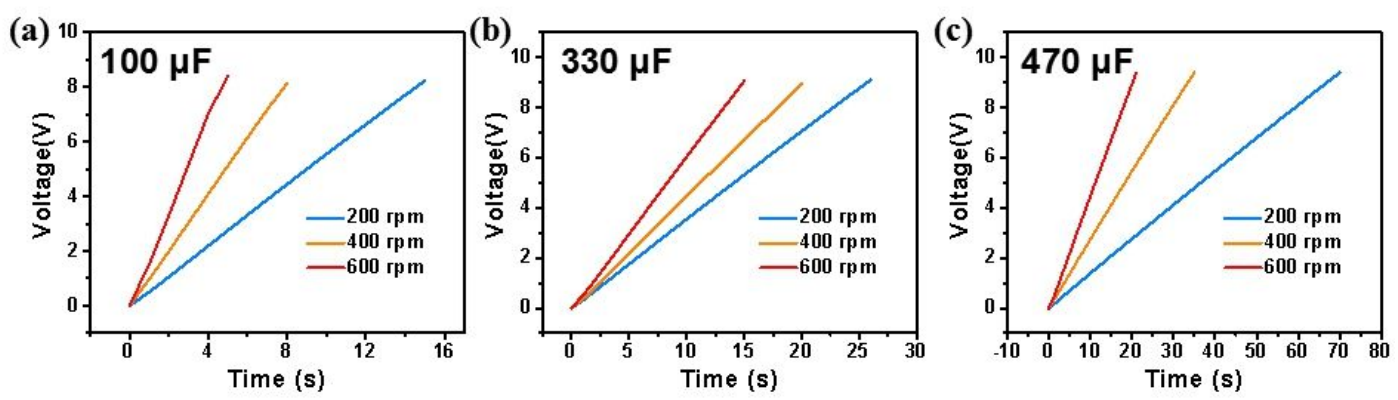

Supplementary Figure 15 The charging curves of capacitors of (a) $100 \mu \mathrm{F}$, (b) 330 $\mu \mathrm{F}$ and (c) $470 \mu \mathrm{F}$ by the lower ER-TENG2 under different rotation speed of motor. 


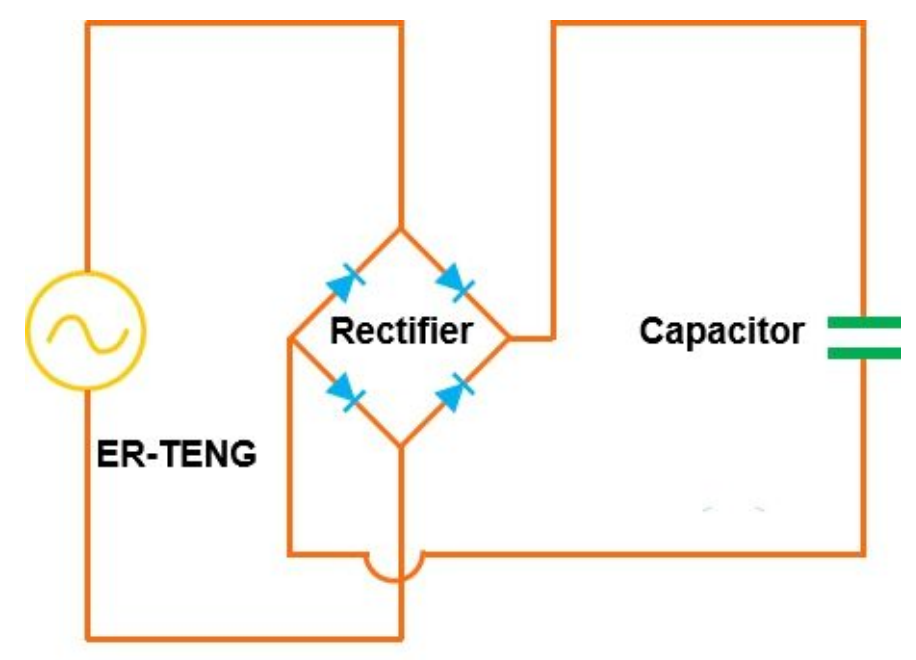

Supplementary Figure 16 The corresponding circuit diagram of ER-TENG to charge capacitors.

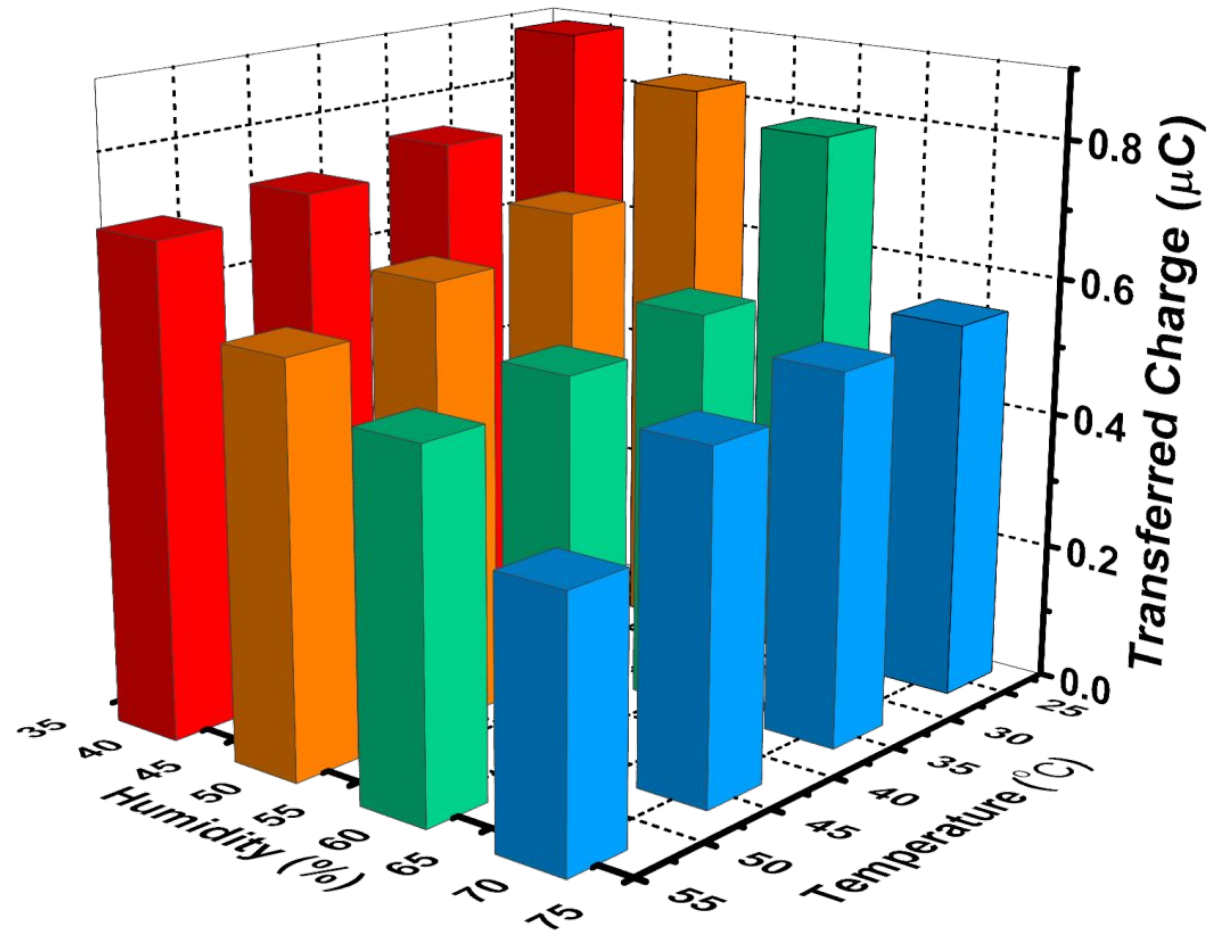

Supplementary Figure 17 The transferred charge of ER-TENG under the different humility and temperature. 


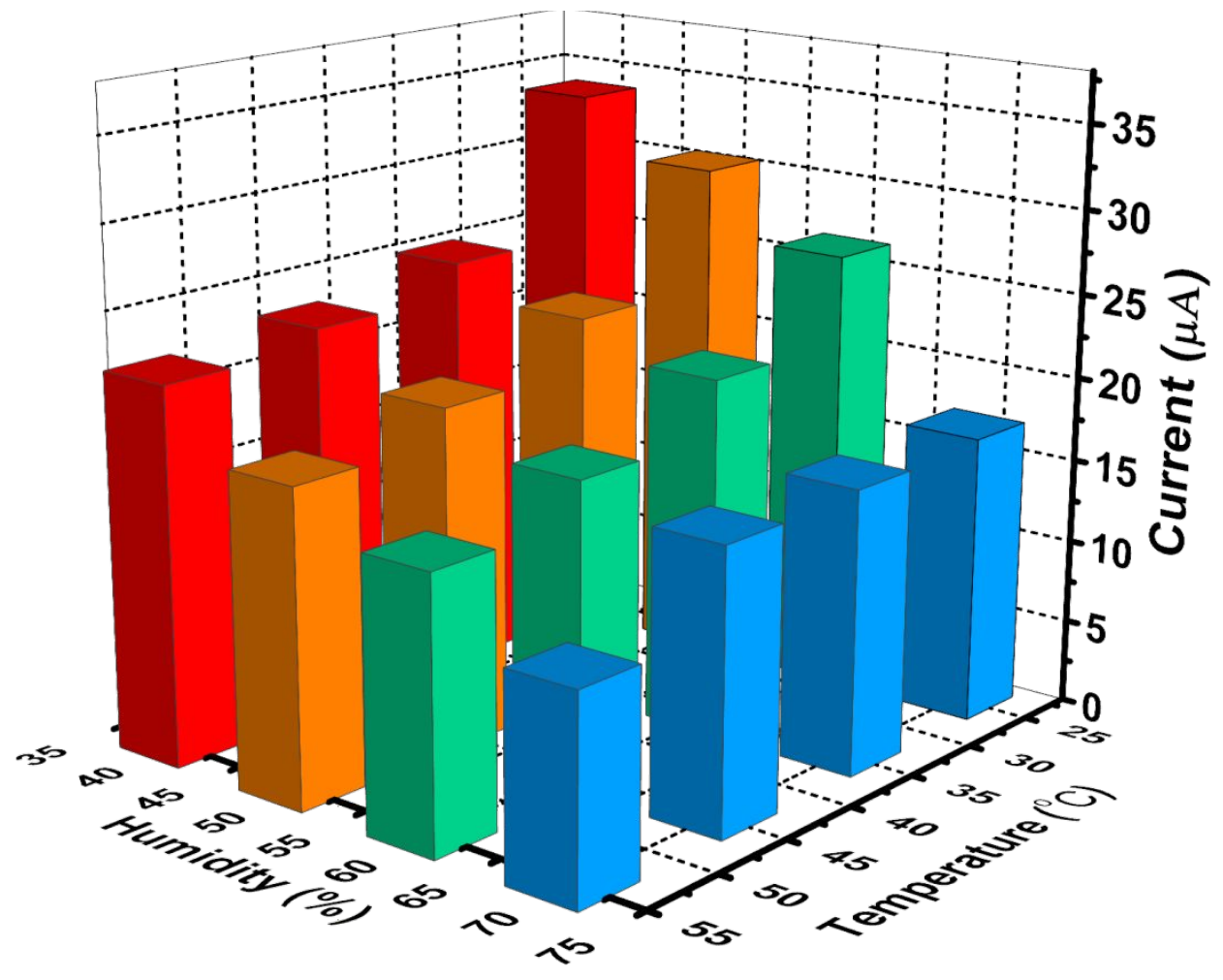

Supplementary Figure 18 The short-circuit current of ER-TENG under the different humility and temperature.

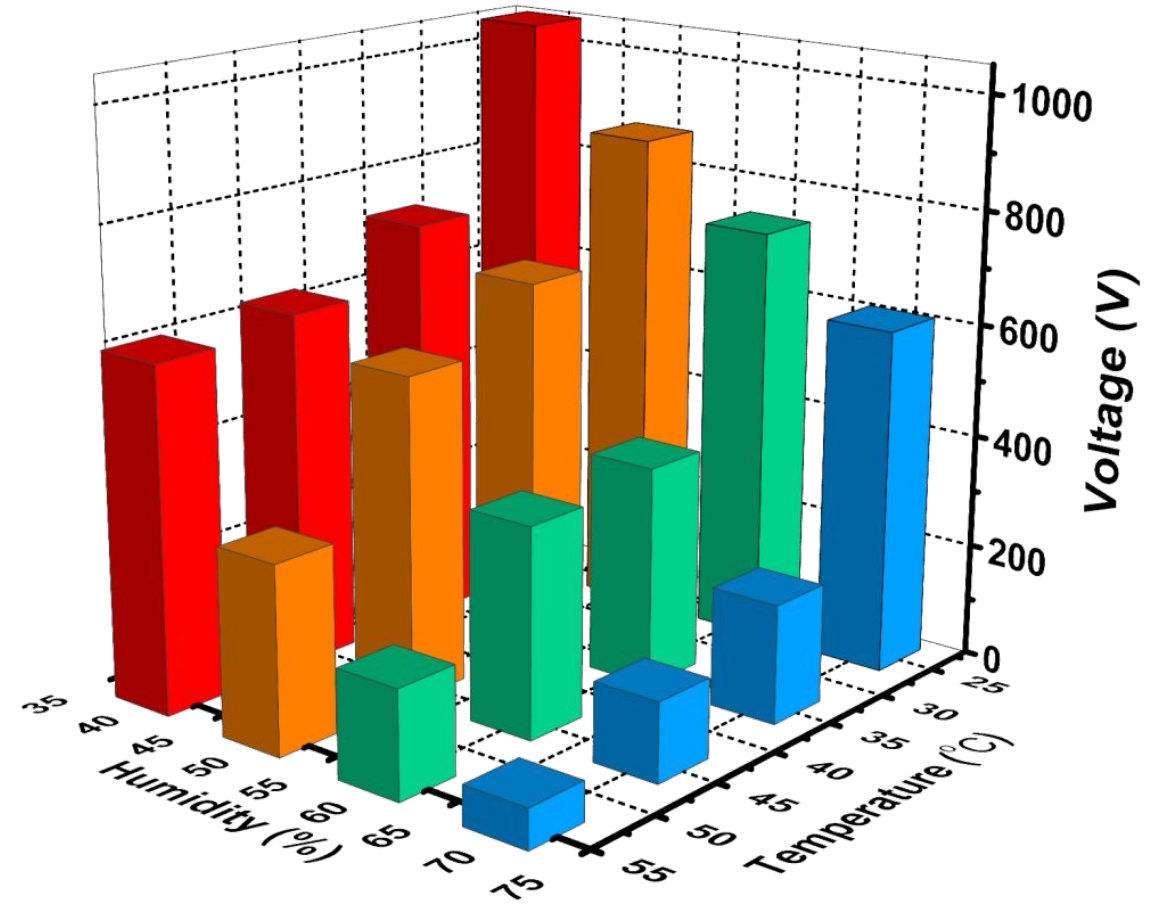

Supplementary Figure 19 The open-circuit voltage of ER-TENG under the different humility and temperature. 


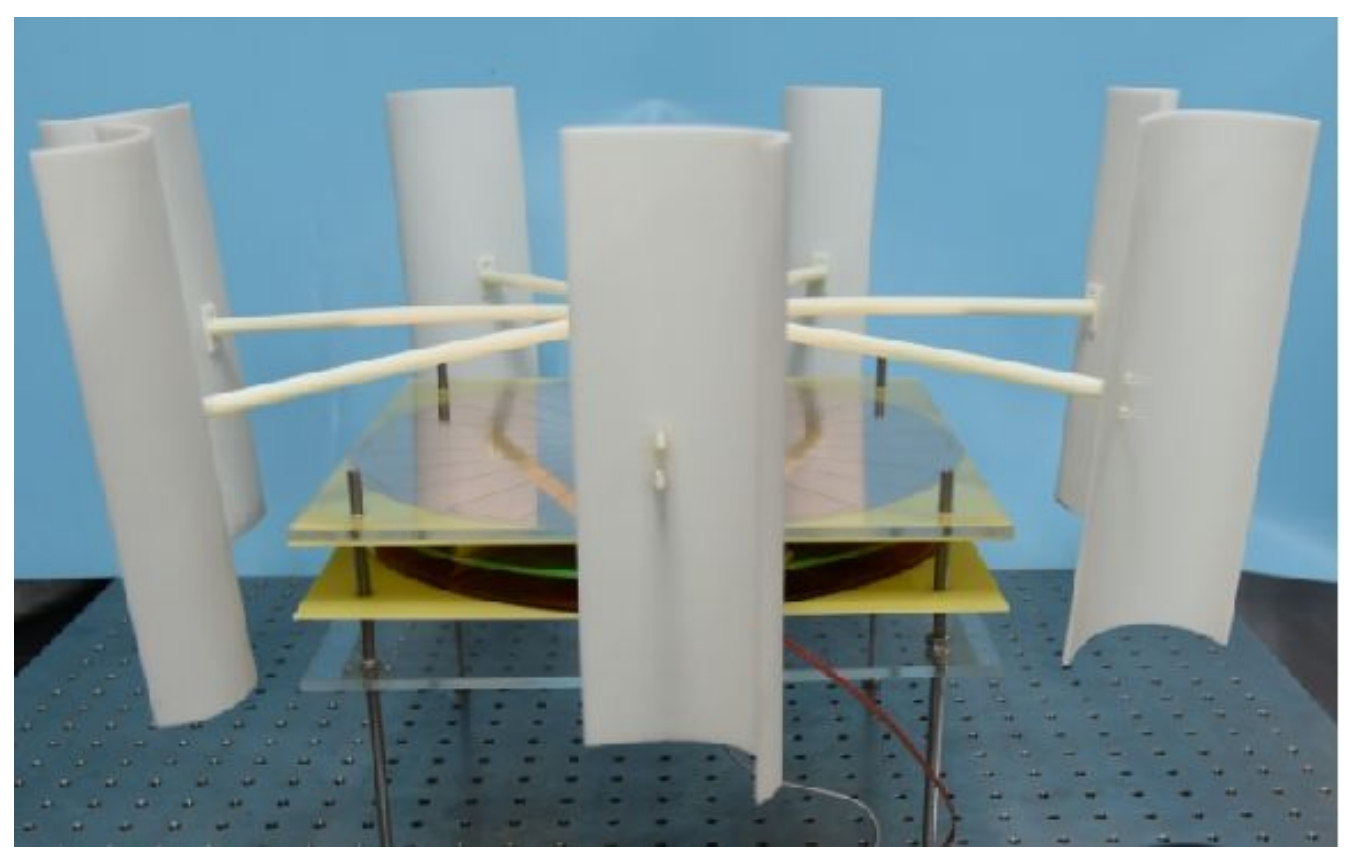

Supplementary Figure 20 The photograph of double-layer ER-TENG with the driving windmill.

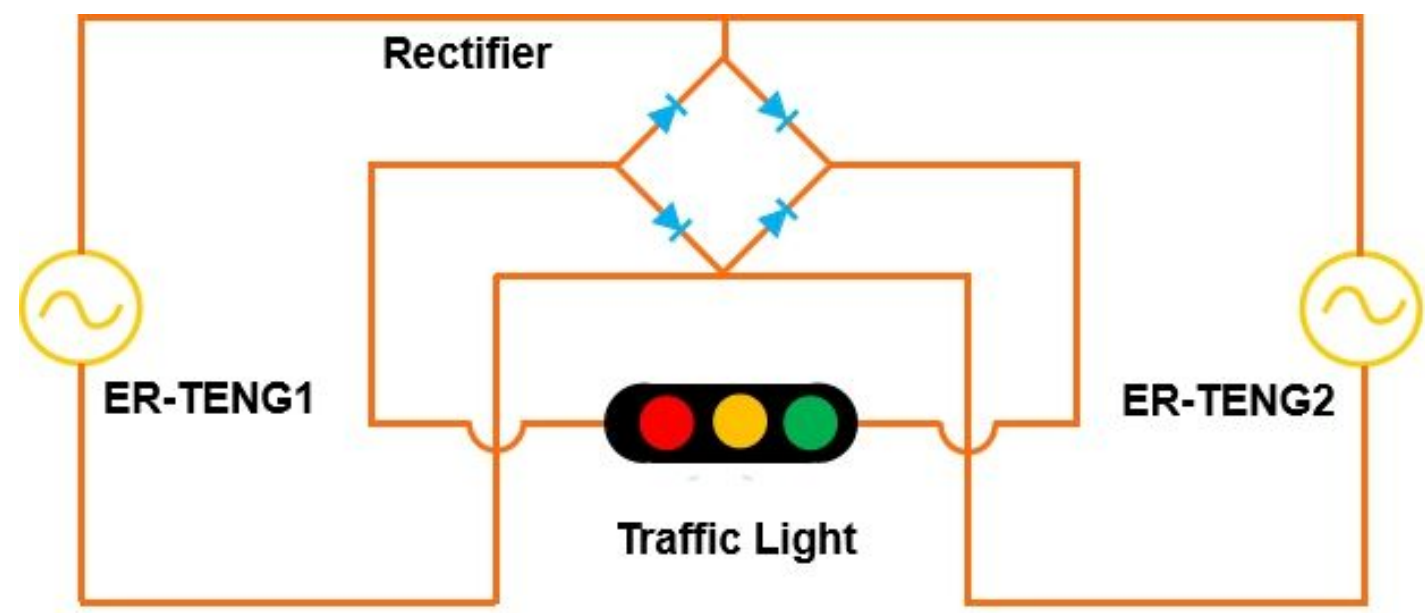

Supplementary Figure 21 The corresponding circuit diagram of double-layer ER-TENG to light the traffic lights. 


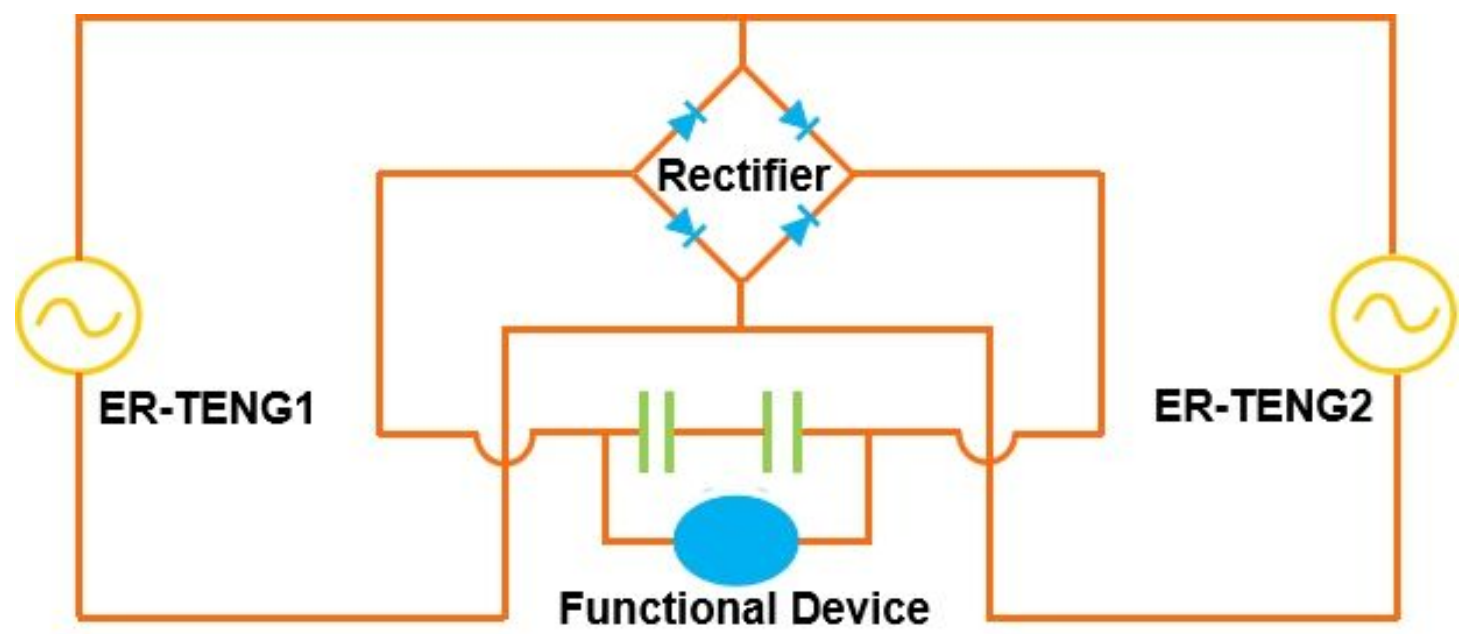

Supplementary Figure 22 The corresponding circuit diagram of double-layer ER-TENG to drive hygrothermograph. 